\section{Novel use of an ultrafiltration device as an alternative method for fluid removal in critically ill pediatric patients with cardiac disease: a case series}

\author{
Sujata Chakravarti, ${ }^{1}$ Yasir Al-Qaqaa, ${ }^{2}$ \\ Meghan Faulkner,1 Puneet Bhatla,1 \\ Michael Argilla, ${ }^{1}$ Michelle Ramirez² \\ 1Division of Pediatric Cardiology; \\ 2Division of Pediatric Critical Care, \\ Department of Pediatrics, New York \\ University School of Medicine, NY, USA
}

\section{Abstract}

Fluid overload (F0) is a common complication for pediatric patients in the intensive care unit. When conventional therapy fails, hemodialysis or peritoneal dialysis is classically used for fluid removal. Unfortunately, these therapies are often associated with cardiovascular or respiratory instability. Ultrafiltration, using devices such as the Aquadex ${ }^{\mathrm{TM}}$ system (Baxter Healthcare, Deerfield, IL, USA), is an effective tool for fluid removal in adult patients with congestive heart failure. As compared to hemodialysis, ultrafiltration can be performed using smaller catheters, and the extracorporeal volume and minimal blood flow rates are lower. In addition, there is no associated abdominal distension as is seen in peritoneal dialysis. Consequently, ultrafiltration may be better tolerated in critically ill pediatric patients. We present three cases of challenging pediatric patients with FO in the setting of congenital heart disease in whom ultrafiltration using the Aquadex ${ }^{\mathrm{TM}}$ system was successfully utilized for fluid removal while cardiorespiratory stability was maintained.

\section{Introduction}

Fluid overload (FO) associated with acute kidney injury (AKI) is common in critically ill pediatric patients, and is associated with poor outcomes.1,2 When conventional therapies fail to achieve a negative fluid balance, early initiation of renal replacement therapy (RRT) has been shown to decrease mortality. 3,4 Traditional modes of RRT, including peritoneal dialysis (PD) and hemodialysis (HD), are highly effective at fluid removal, but are often poorly tolerated in critically ill patients. Several studies have demonstrated advanced ultrafiltration (UF) devices, such as the Aquadex ${ }^{\mathrm{TM}}$ system (Baxter Healthcare, Deerfield, IL,
USA), to be effective at achieving fluid removal while maintaining hemodynamic stability in adults with congestive heart failure.5-9 UF using the Aquadex ${ }^{\mathrm{TM}}$ system can be performed using smaller catheters and the extracorporeal volume and minimal blood flow rates are lower than those required for HD. In addition, there is no associated abdominal distension as is seen in PD. We describe the successful use of the Aquadex ${ }^{\mathrm{TM}}$ system for fluid removal via UF in three critically ill pediatric patients with congenital heart disease.

\section{Case Report \#1}

Case report \#1 is a 11-year-old, $25 \mathrm{~kg}$ male with heterotaxy syndrome of the asplenia type with transposition of the great arteries, unbalanced right dominant atrioventricular canal defect, pulmonary atresia, and total anomalous pulmonary venous connection, who underwent multiple palliative procedures in infancy and subsequently underwent a bidirectional superior cavopulmonary anastomosis at two years of age. Due to persistent pulmonary vein stenosis, he was not felt to be a candidate for a Fontan palliation. The patient presented to our institution with cyanosis. He underwent repair of his atrioventricular canal defect, mitral valve replacement, left ventricle to aortic baffle, and placement of a right ventricle to pulmonary artery conduit. The patient's post-operative course was complicated by a low cardiac output syndrome and tachyarrhythmia causing AKI and acute respiratory failure secondary to significant FO.

After initial treatment with conventional diuretics and fenoldopam failed to produce a negative fluid balance, alternative modalities of fluid removal were considered. Conventional forms of RRT using continuous veno-venous hemofiltration (CVVH) or PD were deemed unsuitable secondary to the patient's tenuous hemodynamic status and high UF needs. UF using the Aquadex ${ }^{\mathrm{TM}}$ system was thought to be feasible due to its low flow capacity. A 6 French, double lumen, reinforced, peripherally inserted central catheter (PICC) was placed in the right lower extremity. After consent was obtained from the parents, UF was initiated. Notably, the patient was anticoagulated with heparin for mechanical valve thromboprophylaxis, and this was adequate for the Aquadex ${ }^{\mathrm{TM}}$ system.

The initial blood flow rate was $10 \mathrm{~mL} /$ minute and this was gradually increased to 40 $\mathrm{mL} / \mathrm{minute}$. UF was started at $10 \mathrm{~mL} /$ hour and was gradually increased. The patient initially experienced mild hypotension requiring fluid administration and a slight increase in vasopressor support. Over the 9-day course of treatment, UF rates were gradually increased to 150
Correspondence: Sujata Chakravarti, Department of Pediatrics, 1Division of Pediatric Cardiology, New York University Langone Medical Center, 560 First Avenue, New York, NY 10016, USA. Tel.: +1.212.263.8352 - Fax: +1.212.263.8301. E-mail: sujata.chakravarti@nyumc.org

Key words: Fluid overload; acute kidney injury; congenital heart disease; ultrafiltration.

Contributions: all authors are accountable for all aspects of this manuscript. SC, conception, data acquisition, drafting, critical revision; YAQ, data acquisition, drafting, critical revision; MF, data acquisition, drafting; PB, critical revision; MA, drafting, critical revision; MR, conception, data acquisition, drafting, critical revision.

Conflict of interest: the authors declare no potential conflict of interest.

Conference presentation: Paper presented at the 45th Critical Care Congress of the Society of Critical Care Medicine, Orlando, Florida, February 2016.

Received for publication: 11 May 2016.

Revision received: 23 May 2016.

Accepted for publication: 30 May 2016.

This work is licensed under a Creative Commons Attribution-NonCommercial 4.0 International License (CC BY-NC 4.0).

(C) Copyright S. Chakravarti et al., 2016 Licensee PAGEPress, Italy

Infectious Disease Reports 2016; 8:6596 doi:10.4081/idr.2016.6596

$\mathrm{mL} /$ hour and the patient tolerated negative fluid balances of greater than $750 \mathrm{~mL} /$ day. There was steady improvement in the patient's clinical condition allowing for weaning of vasopressor and ventilatory support.

Although UF therapy facilitated a significant improvement in the patient's clinical status, he continued to have oliguria. After 9 days of UF, once his hemodynamics had improved, he was transitioned to CVVH. He was ultimately discharged home on PD.

\section{Case Report \#2}

Full term male infant of a diabetic mother with severe hypertrophic cardiomyopathy, respiratory distress syndrome, and persistent pulmonary hypertension of the newborn, who was transferred to our institution with acute respiratory failure. The patient was placed on high frequency oscillatory ventilation, pulmonary vasodilators, and esmolol, but he had persistent severe hypoxemia requiring veno-venous 
extracorporeal membrane oxygenation. The patient had a gradual improvement in respiratory status and he was decannulated after four days. His course was complicated by AKI with F0. Over the next several weeks, the patient was treated with conventional diuretics, fenoldopam, vasopressors, and a peritoneal drain was placed for drainage of ascites. Despite these efforts, a negative fluid balance could not be consistently achieved and FO worsened. PD was not tolerated due to hypoxemia and respiratory acidosis associated with instillation of fluid into the abdomen. Attempts at placement of a HD catheter were unsuccessful, therefore UF using the Aquadex ${ }^{\mathrm{TM}}$ system was considered. A 6 French, double lumen, reinforced, PICC was placed in the inferior vena cava via a transhepatic approach. After consent was obtained, the patient was systemically anticoagulated and UF was initiated.

Blood flow rates were increased from 10 $\mathrm{mL} / \mathrm{minute}$ to $40 \mathrm{~mL} /$ minute without any significant change in hemodynamics. The patient was started at an UF rate of $10 \mathrm{~mL} / \mathrm{hour}$ and this was slowly increased up to $40 \mathrm{~mL} / \mathrm{hour}$ with significant negative fluid balances on the first two days of therapy. The patient subsequently developed thrombocytopenia, hypotension, and elevated lactate due to fungal sepsis, which necessitated discontinuation of UF. The patient later expired despite aggressive medical management.

\section{Case Report \#3}

Female infant with pre-natal diagnosis of Ebstein's anomaly with severe tricuspid regurgitation, pulmonary valve atresia, and significant cardiomegaly with severe lung hypoplasia. The patient was delivered via C-section for hydrops fetalis and non-reassuring fetal heart tracing at 36 weeks gestation. Her post-natal course was complicated by severe hypoxemia and respiratory and metabolic acidosis, as well as atrioventricular re-entrant tachycardia. The patient's condition stabilized with high frequency oscillation ventilation, fluid resuscitation, correctional of metabolic acidosis, inotropic support, vasopressor support, and arrhythmia control. Nonetheless, the patient had AKI with FO despite attempts at diuresis with conventional diuretic therapy and treatment with fenoldopam. Due to declining respiratory status, a peritoneal drain was placed on day of life five with drainage of ascites. PD was attempted, but this was not tolerated due to hypoxemia and hypotension associated with abdominal distension. Consequently, consent was obtained and the decision was made to proceed with UF. Notably, head ultrasound was normal. Patient underwent placement of a 6 French reinforced double lumen PICC via tran- shepatic approach on day of life six. She was placed on low dose heparin and subsequently started on UF therapy using the Aquadex ${ }^{\mathrm{TM}}$ system. The blood flow rate was gradually increased to $35 \mathrm{ml} / \mathrm{min}$ and UF rate was adjusted between 10 and $20 \mathrm{ml} /$ hour. The patient achieved significant negative fluid balances on each of the four days of therapy due to a combination of ultrafiltrate, peritoneal drainage, and increasing urine output. UF was discontinued after four days due to the appearance of intraventricular hemorrhage on head ultrasound. Over the next two weeks, the patient continued to achieve a negative fluid balance with urine and peritoneal output. The parents subsequently elected to withdraw support due to inability to wean respiratory support.

\section{Discussion}

We describe the use of the Aquadex ${ }^{\mathrm{TM}}$ system for UF therapy in three critically ill pediatric patients with FO associated with AKI in the setting of congenital heart disease. AKI, defined as a potentially reversible increase in the blood concentration of creatinine and nitrogenous waste products and by the inability of the kidney to regulate fluid and electrolyte homeostasis, occurs commonly in pediatric patients with critical illness.10 0ne study reported an incidence of $17.9 \%$ in patients admitted to the pediatric intensive care unit, and neonates are particularly susceptible with reported rates as high as $42 \% .11,12 \mathrm{AKI}$ is also common in children following congenital heart surgery with occurrence rates of up to 51$88 \% .13,14$ Despite the fact that it is often reversible, development of AKI is associated with poor outcomes including longer duration of mechanical ventilation, longer intensive care unit length of stay (LOS), and increased mortality. ${ }^{11,15 F 0}$ is frequently a key feature of AKI, and its severity also impacts outcomes.1,2 In fact, FO is itself associated with increased LOS and mortality, even in the absence of documented AKI.16-18 Standard therapy for FO, both in the presence or absence of AKI, includes diuretics, dopaminergic agonists such as fenoldopam to increase renal blood flow, and vasopressor support in an effort to increase renal perfusion pressure.12 When these treatments fail, RRT has traditionally been the only option.

Early initiation of RRT has been associated with decreased mortality in patients with FO secondary to AKI. ${ }^{3,4}$ Options for RRT in critically ill pediatric patients have traditionally included PD and HD. Although PD is frequently effective in reducing FO, its use is limited in certain patients, including those with abdominal diseases, high UF needs, and in children with poor tolerance of abdominal distension due to cardiovascular and/or pulmonary instability. Similarly, while HD, using methods such as $\mathrm{CVVH}$, is highly effective in solute and fluid removal, implementation in critically ill neonates and children can be technically challenging. Typically, a 3 to $4 \mathrm{~kg}$ neonate will require placement of a 6.5 to 7 French HD catheter, and a $25 \mathrm{~kg}$ child would require a 9 to 10 French HD catheter. ${ }^{3}$ In patients with limited options for vascular access, such as those described in this case series, placement of these large bore catheters may not be feasible. In addition, the extracorporeal volume of standard pediatric HD circuits is in excess of 170 $\mathrm{mL}$, which approaches one-half of the total blood volume in neonates. Furthermore, minimal blood flow rates during CVVH are 8 to 12 $\mathrm{mL} / \mathrm{kg} / \mathrm{minute}$ for neonates and 4-6 $\mathrm{mL} / \mathrm{kg} / \mathrm{minute}$ for a child between 15 and 30 kg. ${ }^{3}$ The fluid shifts and flow rates associated with initiation of CVVH are often poorly tolerated in hemodynamically unstable pediatric patients. Since the 1980s, UF has been used as an alternative mechanism of fluid removal in adult patients with congestive heart failure. 5 Numerous clinical trials have demonstrated advanced UF devices, such as the Aquadex ${ }^{\mathrm{TM}}$ system, to be safe and effective, while maintaining hemodynamic stability, avoiding global electrolyte imbalance, and achieving symptomatic relief of congestive heart failure.$^{5-9}$ The less invasive, low flow capability of UF using the Aquadex ${ }^{\mathrm{TM}}$ system makes it an attractive option in patients who may not be able to tolerate more invasive means of fluid removal.

In contrast to CVVH, UF using the Aquadex ${ }^{\mathrm{TM}}$ system can be performed using two 16 gauge reinforced peripheral intravenous catheters or via a 6 French, reinforced, double lumen PICC. This reduces the risks associated with larger catheter insertion into the central vasculature, including infection, hemorrhage, and pneumothorax. ${ }^{9}$ In our first two patients, in whom internal jugular and subclavian access was precluded, in the first case due to his superior cavopulmonary anastomosis, and in the second case due to prior cannulation to extracorporeal membrane oxygenation with sacrifice of the right internal jugular vein, the placement of a 6 Fr PICC was more feasible.

Another potential advantage of the Aquadex ${ }^{\mathrm{TM}}$ system is that the circuit's extracorporeal volume is only $33 \mathrm{~mL}$. Furthermore, UF can be achieved with blood flow rates as low as $10 \mathrm{~mL} / \mathrm{minute}$. These key features minimize rapid intravascular volume shifts and thereby make it an attractive option for fluid removal in patients with unstable hemodynamics. This is supported by the finding that adult patients experience less tachycardia and less variation in systemic vascular resistance during UF $v s$ HD. ${ }^{19}$

The benefits of UF may extend beyond solute and water removal alone. Studies have 
shown that UF membranes, such as those used in the Aquadex ${ }^{\mathrm{TM}}$ system, allow passage of small molecules, including pro-inflammatory cytokines, which may cause myocardial depression and are implicated in the systemic inflammatory response that is observed in infants and children after congenital heart surgery. 5,20 We speculate that removal of these substances by UF in the post-operative period may have contributed to the overall improvement in our first patient's clinical status.

The mechanism of UF is by convection, eliminating iso-osmolar extracellular fluid. A potential benefit is that significant electrolyte abnormalities may be avoided, but until lately, this has precluded the use of the Aquadex ${ }^{\mathrm{TM}}$ system in patients with significant electrolyte derangements or uremia. ${ }^{6,9}$ Askenazi and colleagues recently published their favorable experience using a modified Aquadex ${ }^{\mathrm{TM}}$ machine to perform CVVH in twelve critically ill infants and young children. ${ }^{21}$ Similarly, the CARPEDIEM and Nidus systems have been developed in Europe specifically for the purpose of providing RRT to newborns and young infants with weights ranging from 800 grams to 8 kilograms. All three of these tools offer small extracorporeal circuit volumes, low minimal blood flow rates, and improved UF accuracy. Early reports of the use of these novel devices suggest that the prognosis for this fragile group of patients requiring dialysis may be improving. ${ }^{21,22}$

Similar to CVVH, UF using the Aquadex ${ }^{\mathrm{TM}}$ system requires systemic anticoagulation. As a result, this therapy is contraindicated in patients who are at significant risk of bleeding, and hemorrhagic complications may occur, as was seen in our third patient.

\section{Conclusions}

There are few reports of the use of UF therapy with the Aquadex ${ }^{\mathrm{TM}}$ system in pediatric patients with congenital heart disease. We were successful in achieving a significant net negative fluid balance in a timely fashion in three very complex patients without compromising hemodynamic stability. Further studies are required to assess the utility of this therapy for treatment of FO in a broader population of critically ill pediatric patients.

\section{References}

1. Askenazi DJ, Koralkar R, Hundley HE, et al. Fluid overload and mortality are associated with acute kidney injury in sick nearterm/term neonate. Pediatr Nephrol 2013;28:661-6.

2. Goldstein SL. Advances in pediatric renal replacement therapy for acute kidney injury. Semin Dialysis 2011;24:187-91.

3. Maclaren G, Warwick B. Controversies in paediatric continuous renal replacement therapy. Intensive Care Med 2009;35:596602.

4. Modem V, Thompson M, Gollhofer D, et al. Timing of continuous renal replacement therapy and mortality in critically ill children. Pediatr Crit Care 2013;42:943-53.

5. Bart BA, Boyle A, Bank AJ, et al. Ultrafiltration versus usual care for hospitalized patients with heart failure: the relief for acutely fluid-overloaded patients with decompensated congestive heart failure (RAPID-CHF) trial. J Am Coll Cardiol 2005;46:2043-6.

6. Emery RW, Hommerding J, Emery AM, et al. Use of peripheral ultrafiltration in the postoperative cardiac surgery patient. Innovations 2007;2:33-5.

7. Costanzo MR, Saltzberg MT, O'Sullivan J, Sobotka P. Early ultrafiltration in patients with decompensated heart failure and diuretic resistance. J Am Coll Cardiol 2005;46:2047-51.

8. Costanzo MR, Saltzberg MT, Jessup M, et al. Ultrafiltration is associated with fewer rehospitalizations than continuous diuretic infusion in patients with decompensated heart failure: Results from UNLOAD. J Card Fail 2010;16:277-84.

9. Costanzo MR, Negoianu D, Jaski BE, et al. Aquapheresis versus intravenous diuretics and hospitalizations for heart failure. JACC Heart Fail 2016;4:95-105.

10. Andreoli SP. Acute kidney injury in children. Pediatr Nephrol 2009;24:253-63.

11. Alkandari 0, Eddington KA, Hyder A, et al. Acute kidney injury is an independent risk factor for pediatric intensive care unit mortality, longer length of stay and prolonged mechanical ventilation in critically ill children: a two center retrospective cohort study. Crit Care 2011;15: R146.
12. Selewski DT, Charlton JR, Jetton JG, et al. Neonatal acute kidney injury. Pediatrics 2015;136:e463.

13. Aydin SI, Seiden HS, Blaufox AD, et al. Acute kidney injury after surgery for congenital heart disease. Ann Thorac Surg 2012;94:1589-95.

14. Mamikonian LS, Mamo LB, Smith PB, et al. Cardiopulmonary bypass is associated with hemolysis and acute kidney injury in neonates, infants, and children. Pediatr Crit Care Med 2014;15:e111-9.

15. Shalaby M, Khathlan N, Safder 0, et al. Outcome of acute kidney injury in pediatric patients admitted to the intensive care unit. Clin Nephrol 2014;82:379-86.

16. Bhaskar P, Dhar AV, Thompson M, et al. Early fluid accumulation in children with shock and ICU mortality: a matched casecontrol study. Intensive Care Med 2015;41: 1445-53.

17. Ketharanathan N, McCulloch M, Wilson C. Fluid overload in a South African pediatric intensive care unit. J Tropical Pediatr 2014;60:428-33.

18. Sampaio TZ, O'Hearn K, Reddy D, Menon $K$. The influence of fluid overload on the length of mechanical ventilation in pediatric congenital heart surgery. Pediatr Cardiol 2015;36:1692-9.

19. Fox SD, Henderson LW. Cardiovascular response during hemodialysis and hemofiltration: thermal, membrane, and catecholamine influences. Blood Purif 2013;11:224-36.

20. Journois D, Pouard P, Greeley WJ, et al. Hemofiltration during cardiopulmonary bypass in pediatric cardiac surgery. Anesthesiology 1994;81:1181-9.

21. Askenazi D, Ingram D, White $S$, et al. Smaller circuits for smaller patients: improving renal support therapy with Aquadex $^{\text {TM }}$. Pediatr Nephrol 2016;31:85360.

22. Hothi D. Designing technology to meet the therapeutic demands of acute renal injury in neonates and small infants. Pediatr Nephrol 2014;29:1869-71. 